\title{
Editorial
}

\section{Data Assimilation in Numerical Weather and Climate Models}

\author{
Shaoqing Zhang, ${ }^{1}$ Guijun Han, ${ }^{2}$ Yuanfu Xie, ${ }^{3}$ and Juan Jose Ruiz ${ }^{4}$ \\ ${ }^{1}$ Geophysical Fluid Dynamics Laboratory, NOAA, Princeton, NJ 08542, USA \\ ${ }^{2}$ National Marine Data and Information Service, State Oceanic Administration, Tianjin 300171, China \\ ${ }^{3}$ Earth System Research Laboratory, NOAA, Boulder, CO 80305, USA \\ ${ }^{4}$ University of Buenos Aires, Viamonte, 4301053 Buenos Aires, Argentina
}

Correspondence should be addressed to Shaoqing Zhang; shaoqing.zhang@noaa.gov

Received 2 June 2015; Accepted 9 June 2015

Copyright (C) 2015 Shaoqing Zhang et al. This is an open access article distributed under the Creative Commons Attribution License, which permits unrestricted use, distribution, and reproduction in any medium, provided the original work is properly cited.

Great efforts have been made in climate modeling in the past decades. While a coupled model can simulate the interaction of the atmosphere, ocean, ice, and land and assess the fundamental feature of climate evolution, modeling on weather phenomena has been progressively advanced. The model resolution is higher and higher and the model physics are more and more accurate. Now, seamless weatherclimate models are emerging. One expects more extensive applications of weather-climate models in environment monitoring and forecasting. Assessing instantaneous variability and providing accurate initial conditions for model forecasts require scientifically incorporating instrumental data into numerical model systems. This process is data assimilation.

Data assimilation uses model dynamics and physics to extract observational information from measured data which are usually scattered in time and space. The modern data assimilation pursues balanced and coherent state analysis and estimation and has grown as an important branch field in earth sciences. Not only does a good data assimilation product provide initial conditions for weather-climate predictions, but it serves also as a basis for one to further understand the mechanisms of weather-climate development, through reconstructing the continuous time series of weather and climate variables with three-dimensional structures. Furthermore, derived from information estimation theory, data assimilation develops out an important topic-how to use observational information to optimize model parameters, called "parameter estimation." Parameter estimation helps modelers more accurately understand the sensitivity of model parameters and more objectively determine the values of parameters. We can predict that data assimilation will become a more important field in the near future due to the demanding of the society on the high quality of environment monitoring and forecasting.

In this special issue, we collect 13 papers that cover observing system assessment and impact (3), weather-climate analysis and prediction (5), analysis and model mechanism studies (2), recent advances in data assimilation methods (1), and parameter estimation (1), as well as ocean safety study (1). This collection includes most of the major topics in modern data assimilation. We expect that, through this special issue, more scientists recognize the importance of data assimilation in weather-climate modeling and predictions. While a high quality weather-climate forecast makes more societal significance, as the return, it helps advance the earth science.

Observing system assessment and impact is an important branch field in data assimilation. In the paper "Impact of Argo Observation on the Regional Ocean Reanalysis of China Coastal Waters and Adjacent Seas: A Twin-Experiment Study," the authors design a twin experiment to study the Argo impact on regional ocean reanalysis, providing an insight on importance of Argo for coastal areas. Nowadays, satellite observations play more and more important roles in numerical weather prediction. Assessing data quality is crucial for the best use of them. The paper "An Assessment of Data from the Advanced Technology Microwave Sounder at the Met Office" addresses this concern for ATMS data by comparing their departure from UK Met Office forecasts to AMSU and MHS satellite. It provides a complimentary validation of the data quality to the assessment at ECMWF for improving prediction of severe weather events. Tropospheric Airborne Meteorological Data Reporting (TAMDAR) has been providing a continuous operational stream 
of real-time observations for regional weather forecasts. In the paper "Using Adjoint-Based Forecast Sensitivity Method to Evaluate TAMDAR Data Impacts on Regional Forecasts," an adjoint-based Forecast Sensitivity to Observation (FSO) method is used to evaluate the impact of TAMDAR observations on regional 24-hour forecast error reduction over the Continental United States domain. Compared to the traditional observation-denial method, often referred to as Observation System Experiments (OSEs), the FSO method is advantageous due to its efficiency on the impact estimation for a complete set of observations, or any subset of observations grouped by type of observing system, observed variable, geographic region, vertical level, or other categories.

An important issue in data assimilation is how to deal with the multiscale nature of ocean and atmospheric dynamics. In the paper "Diffusion Filters for Variational Data Assimilation of Sea Surface Temperature in an Intermediate Climate Model," a gradient diffusion filter technique is introduced to reconstruct the multiscale variability of a scalar field from a nonuniform observation network. The approach successfully recovers large scale patterns over the entire domain and accurately reconstructs small scale features over densely observed regions. This methodology has been successfully tested with a simplified coupled general circulation model producing a positive impact upon the representation of the oceanic and atmospheric flow.

Reanalysis data sets of the ocean and the atmosphere are extremely valuable tools for the study of physical processes and the variability of the system at different spatial and time scales. In the paper entitled "Low-Frequency Variability of the Yellow Sea Cold Water Mass Identified from the China Coastal Waters and Adjacent Seas Reanalysis," the interannual and decadal variability of the yellow sea temperature is analyzed and related to the known coupled variability modes (i.e., Artic Oscillation, El Niño/Southern Oscillation, and Pacific Decadal Oscillation) in order to advance in the understanding of the physical processes that drive the climatology of the Yellow Sea. The paper "Statistical Prediction of the South China Sea Surface Height Anomaly" combines an ocean data assimilation product and statistics to explore the forecast of South China Sea surface height, providing a method for local ocean variable forecast. The papers "Research on Cold Core Eddy Change and Phytoplankton Bloom Induced by Typhoons: Case Studies in the South China Sea" and "Upper Ocean Thermal Responses to Sea Spray Mediated Turbulent Fluxes during Typhoon Passage" investigate the influences of tropical cyclones on eddyassociated phytoplankton bloom and sea spray associated turbulent fluxes, providing us a new view angle to understand the impact of typhoon on local ocean states. One societal impact of severe weather is heavy precipitation causing flooding. The paper "Prediction of Moderate and Heavy Rainfall in New Zealand Using Data Assimilation and Ensemble" investigates the moderate and heavy rainfall events in New Zealand. The authors' experience with these severe weather events in New Zealand could help us better predict heavy rainfall events at complex terrain with observations and by more sophisticated ensemble methods.
The impact of parameter uncertainties on the results of data assimilation can be studied using sensitivity analysis. In the paper "Some Aspects of Sensitivity Analysis in Variational Data Assimilation for Coupled Dynamical Systems," a sensitivity analysis method, based on the theory of shadowing pseudoorbits in dynamical systems, is applied under the framework of four-dimensional variational data assimilation (4D-Var) to a low-order coupled nonlinear dynamical system composed of fast and slow versions of the Lorenz model. Results demonstrated that this proposed sensitivity analysis method is more efficient compared with the conventional method. The paper "A Study of Coupling Parameter Estimation Implemented by $4 \mathrm{D}$-Var and EnKF with a Simple Coupled System" investigates the difference performance of 4D-Var and EnKF parameter estimation using cross-media observations in coupled systems. Although the study was carried out within a simple coupled system, it provides guidance for coupling parameter estimation.

The paper "Initialized Decadal Predictions by LASG/IAP Climate System Model FGOALS-s2: Evaluations of Strengths and Weaknesses" provides a thorough statistical evaluation of a decadal prediction system, which is meaningful for us to understand some aspects of decadal prediction significances. In addition, in the paper "An Evaluation Method of Underwater Ocean Environment Safety Situation Based on D-S Evidence Theory," authors present an evaluation method for underwater environment safety situation based on the Dempster-Shafer evidence theory. In terms of ocean engineering, this study provides a new view angle for oceanographer to understand the ocean safety.

Shaoqing Zhang
Guijun Han
Yuanfu Xie
Juan Jose Ruiz



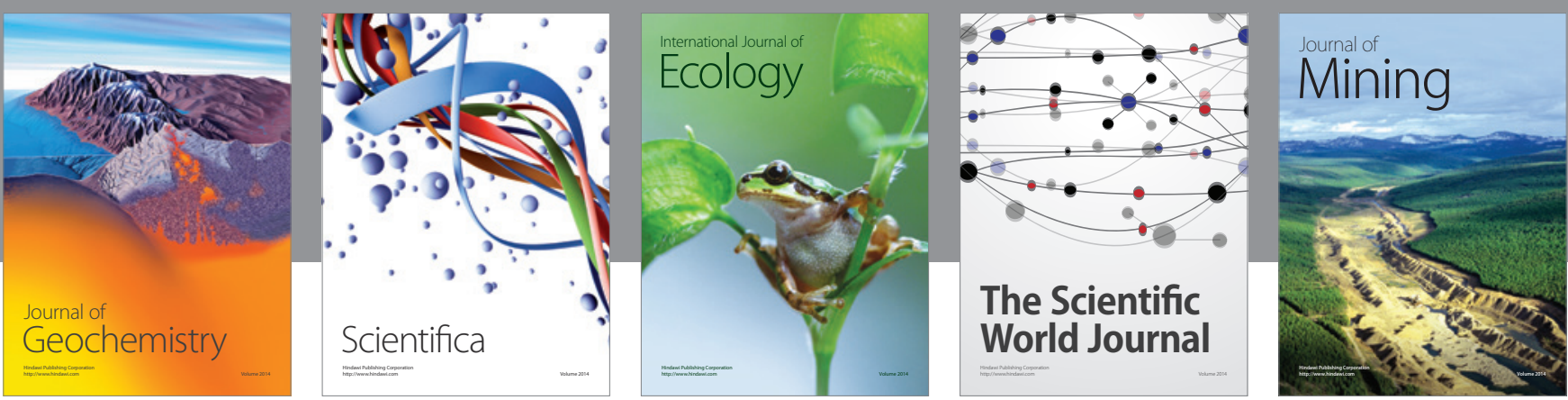

The Scientific World Journal
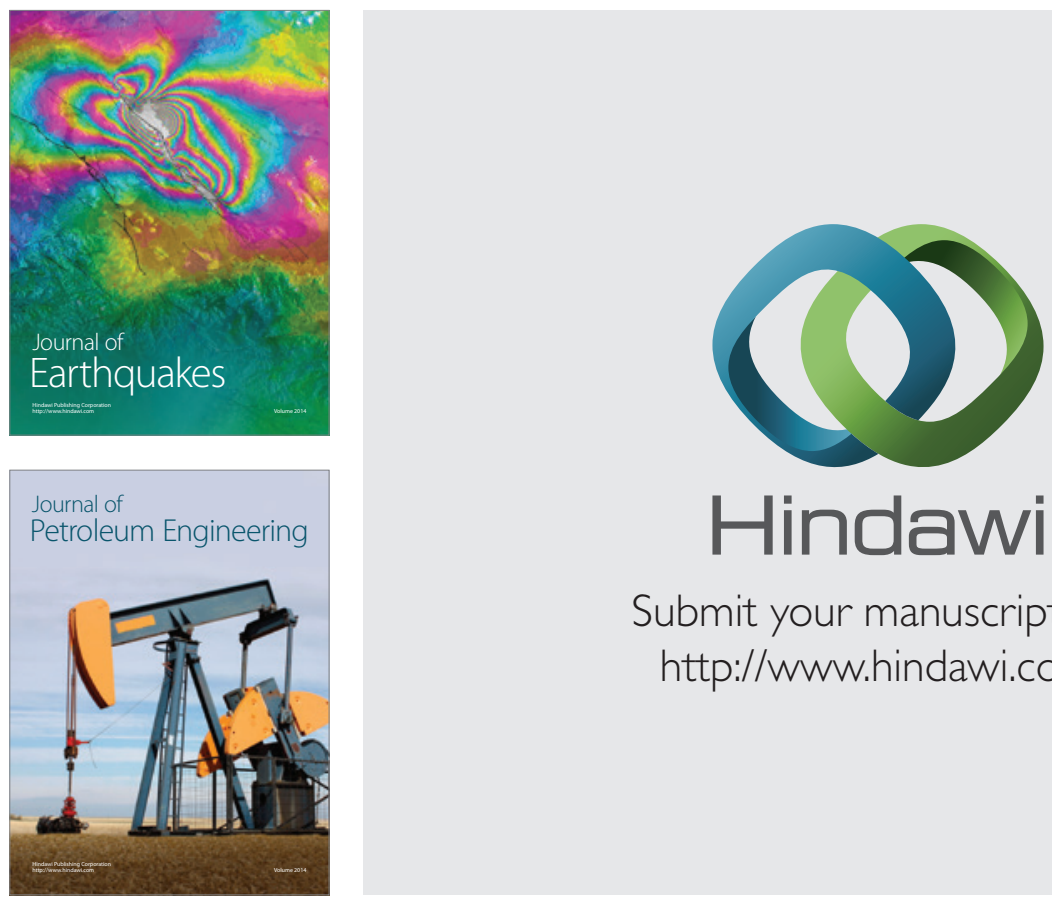

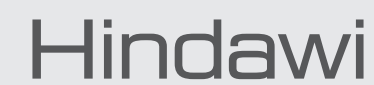

Submit your manuscripts at

http://www.hindawi.com
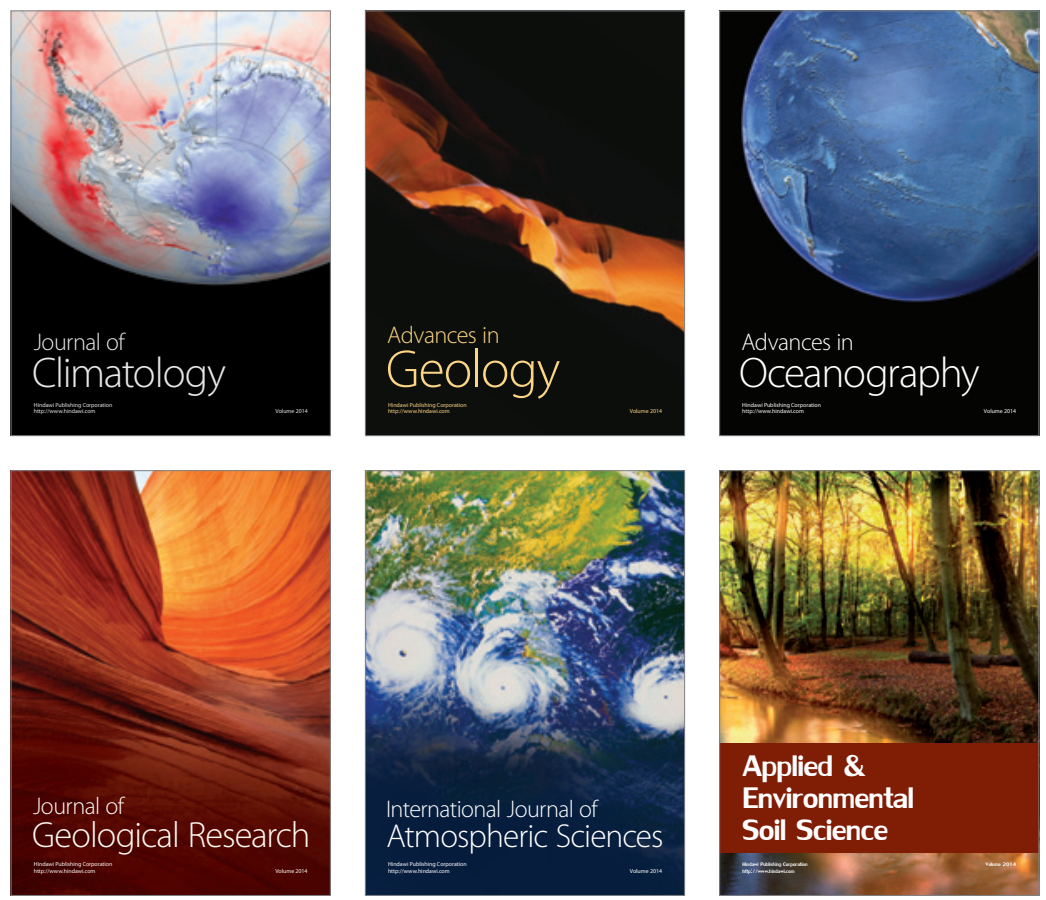
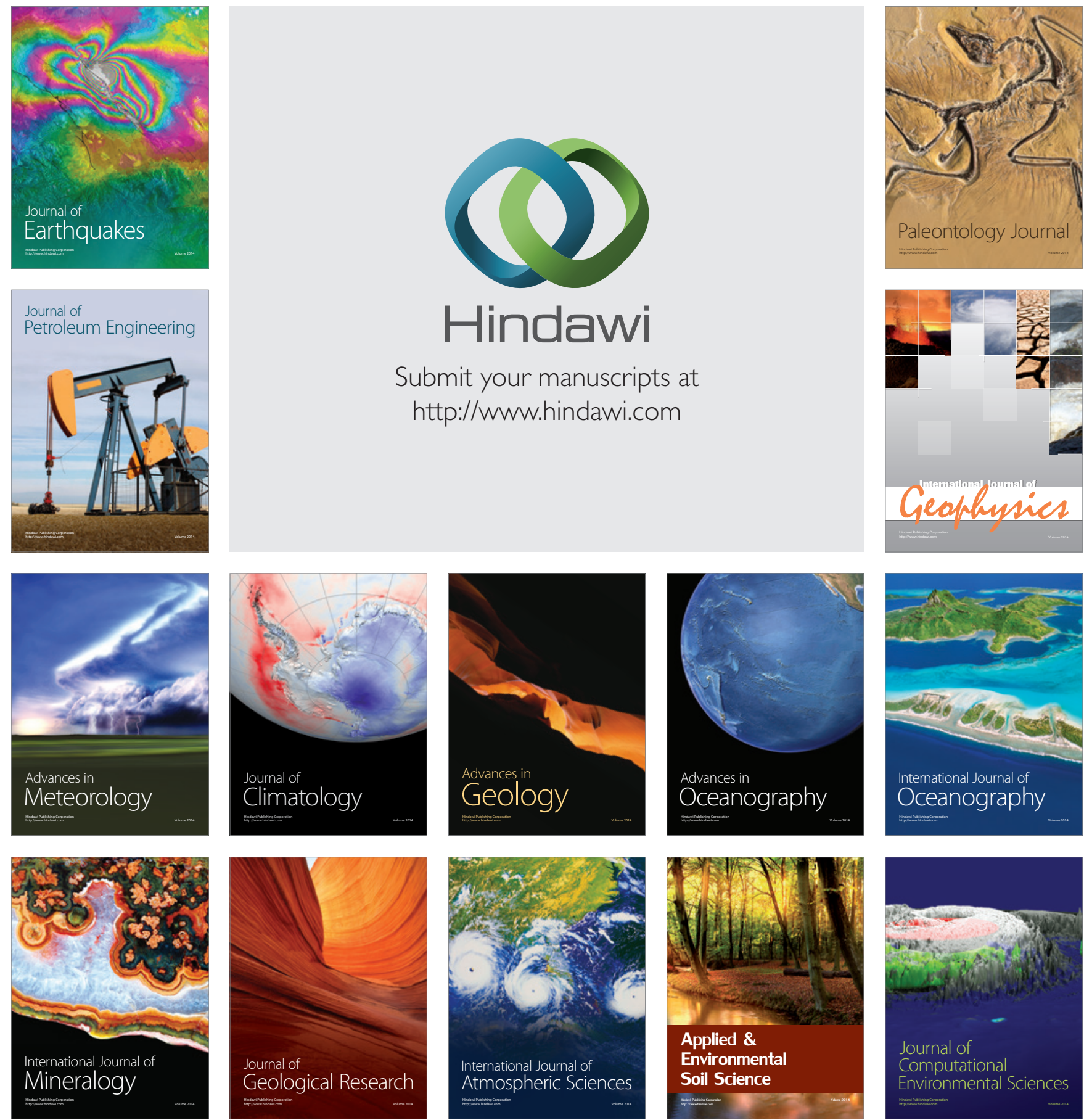